\title{
Prevalence of Chlamydia trachomatis infection in parturient women in Gipuzkoa, Northern Spain
}

\author{
Luis Piñeiro ${ }^{1}$, Arantza Lekuona ${ }^{2}$, Gustavo Cilla ${ }^{1,3^{*}}$, Izaskun Lasa², Laura-Pilar Martinez-Gallardo², Javier Korta ${ }^{4}$ \\ and Emilio Pérez-Trallero 1,3,5
}

\begin{abstract}
The prevalence of Chlamydia trachomatis infection in Southern Europe is poorly understood and its identification is essential for the design of appropriate prevention policies. The prevalence of C. trachomatis in 2011-2014 was determined through polymerase chain reaction in urine samples from 11,687 unselected parturient women from the Basque Country, Spain (San Sebastián area). The overall age-adjusted prevalence was $1.0 \%$ (95 \% Cl 0.8-1.2). The prevalence of infection in women younger than 25 years was $6.4 \%$ and decreased substantially with increasing age: $2.0 \%$ in $25-29$ year-olds and $0.5 \%$ in older women $(P<0.001)$. The prevalence was higher in parturient of foreign origin (1.9\%, $95 \% \mathrm{Cl} 1.3-2.5)$ than in Spanish parturients $(0.8 \%, 95 \% \mathrm{Cl} 0.6-1.0),(P<0.001)$. The results of this study support the need to screen young women as part of antenatal care in Spain.
\end{abstract}

Keywords: Chlamydia trachomatis, Prevalence, Pregnant women, Parturient women, Asymptomatic women, Spain

\section{Background}

Chlamydia trachomatis (C. trachomatis) is the most diagnosed sexually transmitted bacterial infection worldwide, with an estimated 100 million new infections each year (WHO 2012). Chlamydial infections in women frequently cause cervicitis and urethritis, but it has been estimated that up to $70-75 \%$ of the cases are asymptomatic. Asymptomatic infection facilitates its spread to sexual contacts and can cause serious sequelae, including pelvic inflammatory disease, tubal damage, ectopic pregnancy, and infertility (Cates and Wasserheit 1991; Haggerty et al. 2010). In pregnant women, the infection has been associated with adverse pregnancy outcomes such as prematurity, and may be transmitted vertically to the newborn, which may result in neonatal ocular or respiratory tract infection (Blas et al. 2007; Rours et al. 2011; Hammerschlag 2011).

\footnotetext{
*Correspondence: carlosgustavosantiago.cillaeguiluz@osakidetza.eus 1 Microbiology Department, Hospital Universitario Donostia, Paseo Dr Beguiristain s/n, 20014 San Sebastián, Spain

Full list of author information is available at the end of the article
}

The knowledge of the prevalence of the C. trachomatis infection is essential for the design of appropriate infection control programs. In most countries of Southern Europe, the prevalence of $C$. trachomatis infection in pregnant women is not well characterized. Moreover, even though these women are a potential target group for prevention policies (ECDC 2014; CDC et al. 2014; Böhm et al. 2009), to date no recommendation has been made in Spain in this regard. The aim of this study was to estimate the prevalence of $C$. trachomatis infection in an unselected population of pregnant women at term, by age-group and geographical area of origin, in the Basque Country, Spain.

\section{Methods}

The study was conducted in the city of San Sebastián and surrounding counties (413,000 inhabitants, with 4000-4500 deliveries per year). Eighty-seven percent of parturient women in the area attended Donostia University Hospital, the remainder attended private health care facilities.

The presence of C. trachomatis was determined in urine samples (first catch) collected from unselected parturient 
women who delivered a live neonate in the Donostia University Hospital between January 2011 and December 2014. C. trachomatis determination was included in a previously established free-of-charge prevention program for sexually-transmitted diseases in our hospital, which included serological detection of Treponema pallidum and human immunodeficiency virus (HIV). Women were informed about $C$. trachomatis infections and were screened in the immediate postpartum period, unless they refused to participate. Samples were preserved at $4{ }^{\circ} \mathrm{C}$ until processing, which was carried out within $48 \mathrm{~h}$. C. trachomatis DNA was detected using a real-time PCR (Cobas ${ }^{\circledR} 4800$ CT/NG, Roche Diagnostics, Branchburg, NJ, USA), which also detects Neisseria gonorrhoeae DNA. Samples with an invalid result (no amplification of the internal control) were diluted appropriately and were re-analyzed. Detection of antibodies against Treponema pallidum and HIV was performed with a chemiluminescent microparticle immunoassay (CMIA) (Architect Syphilis TP reagent, and Architect HIV Ag/Ab Combo, Abbott Laboratories, Wiesbaden, Germany). Treponema CMIA-positive samples were reanalyzed using fluorescent treponemal antibody absorption (FTA-Abs) (BioMèrieux, Marcy l'Ètoile, France) and Venereal Disease Research Laboratory (VDRL) (Siemens, Marburg, Germany) tests. Upon detection of C. trachomatis infection, women were referred to a gynecologist for treatment (azithromycin $1 \mathrm{~g}$ orally), as well as treatment of their sexual partners, if applicable. Similarly, their newborns were placed under medical and microbiological surveillance (medical examination and pharyngeal exudate 7-10 days after birth).

The prevalence of infection and its $95 \%$ exact binomial confidence interval (CI) were calculated based on the number of Chlamydia-positive women (numerator) and on the number of women with a valid result of the diagnostic test (denominator). To estimate the prevalence according to the women's geographical origin (native or foreign immigrant), the country of origin in all Chlamydia-positive women and in a sample of 1124 parturient women of the study was identified. As the proportion of immigrants vary widely with age, this sample was chosen by stratified random sampling in the four age-groups in which was divided the population of the study (sample size estimated with $5 \%$ precision and $95 \%$ confidence interval for each age-group) using as reference population that of parturient women from the Basque Country classified by age and country of origin, provided by the Basque Institute of Statistics. The distribution by countries found in the 1124 women was extrapolated to the population of women investigated. The overall prevalences were age-adjusted in relation to the distribution by the age of the total number of parturients in the area
(Basque Institute of Statistics). The Chi square test was used to compare percentages with application of Fisher's corrections (two-tailed), when required. A $P$ value of $<0.05$ was considered statistically significant. The study was approved by the Ethics Committee for Clinical Research of the Health Area of Gipuzkoa.

\section{Results}

Samples were analysed from 12,228 parturient women (median age 33 years, range 14-54), representing 76.4 \% of the eligible population screened $(84.6 \%$ in the last three years). The result was invalid (inhibition of internal control amplification) in 541/12,228 samples (4.4\%), corresponding mostly to haematuric urine samples. $C$. trachomatis was detected in 121/11,687 women, with an overall age-standardized prevalence of $1 \%$ (95\% CI 0.8 1.2) and an annual prevalence ranging from $1.2 \%$ in 2011 to $0.8 \%$ in 2013 ( $P=0.14$, non-significant). The median age of $C$. trachomatis-positive women was 27 years, range $17-43$.

The prevalence of infection was higher in younger women, being $6.4 \%$ in those aged $<25$ years, and decreased with increasing age: $2.0 \%$ in 25-29 yearolds and $0.5 \%$ in women aged $\geq 30$ years old $(P<0.001)$ (Table 1 ). Women aged $<25$ and $<30$ years old represented 5.1 and $20.5 \%$ of parturient women but accounted for 31.4 and $60.3 \%$ of detected cases, respectively. Overall and in all age groups, prevalence was higher in immigrant than in Spanish parturient women [overall age-standardized prevalence $1.9 \%$ (95 \% CI 1.3-2.5) vs. 0.8 \% (95\% CI 0.6-1.0) respectively, $P<0.001$ ] (Table 1 ). Parturient women of Latin American origin had the highest prevalence $(4.9 \%)$ and they also were the main group of population among immigrants (43\%), accounting for $37.2 \%$ of all infected women and $78.9 \%(n=45)$ of infected immigrants. The estimated prevalence in women of North African origin, the second largest immigrant group in Spain, was $1 \%$ in this study $(4 / 418)$.

Neither Neisseria gonorrhoeae nor current Treponema pallidum infection (VDRL positive) was detected in any of the 11,687 parturient women, although 27 women (0.2\%) (one of whom was Chlamydia-infected) had a history of syphilis (CMIA/FTA-Abs positive). Seven women $(0.1 \%)$ had HIV infection but none of these was coinfected with C. trachomatis. Only two Chlamydia-positive patients had symptoms attributable to the infection during pregnancy (one woman with cervicitis at week 25 and another with dysuria, leukocyturia and negative urine culture in week 36). A further four women had nonspecific symptoms of doubtful interpretation (vulvovaginal pruritus and/or vaginal discharge). The prevalence of preterm birth (neonates less than 37 weeks of gestational age) in Chlamydia-positive mothers was $7.6 \%(\mathrm{n}=9)$. 
Table 1 Prevalence of $C$. trachomatis infection by age and country of origin in puerperal women from the San Sebastián area, Basque Country, Spain (2011-2014)

\begin{tabular}{|c|c|c|c|c|c|c|c|c|c|c|c|c|c|}
\hline \multirow[t]{2}{*}{ Age $^{\mathrm{a}}$ (years) } & \multicolumn{4}{|l|}{ Total } & \multicolumn{4}{|c|}{ Native } & \multicolumn{4}{|c|}{ Foreign immigrants } & \multirow[t]{2}{*}{$P$} \\
\hline & No. & $\mathrm{CT}+$ & (\%) & $(95 \% \mathrm{Cl})$ & No. ${ }^{b}$ & $\mathrm{CT}+$ & (\%) & $(95 \% \mathrm{Cl})$ & No. ${ }^{b}$ & $\mathrm{CT}+$ & (\%) & $(95 \% \mathrm{Cl})$ & \\
\hline$<25$ & 596 & 38 & $(6.4)^{c}$ & $(4.7-8.6)$ & 247 & 14 & $(5.7)$ & $(3.4-9.3)$ & 349 & 24 & (6.9) & $(4.7-10.0)$ & 0.552 \\
\hline $25-29$ & 1799 & 35 & $(2.0)$ & $(1.4-2.7)$ & 1164 & 16 & $(1.4)$ & $(0.9-2.2)$ & 635 & 19 & (3.0) & $(1.9-4.6)$ & 0.018 \\
\hline $30-34$ & 4710 & 26 & $(0.6)$ & $(0.4-0.8)$ & 4065 & 18 & $(0.4)$ & $(0.3-0.7)$ & 645 & 8 & $(1.2)$ & $(0.6-2.4)$ & $0.019^{c}$ \\
\hline$\geq 35$ & 4582 & 22 & $(0.5)$ & $(0.3-0.7)$ & 4073 & 16 & $(0.4)$ & $(0.2-0.6)$ & 509 & 6 & $(1.2)$ & $(0.6-2.5)$ & $0.029^{\circ}$ \\
\hline Total & 11,687 & 121 & $(1.0)^{\mathrm{e}}$ & $(0.9-1.2)$ & 9549 & 64 & $(0.7)^{e}$ & $(0.5-0.8)$ & 2138 & 57 & $(2.7)^{e}$ & $(2.1-3.4)$ & $<0.001$ \\
\hline
\end{tabular}

a Median age 33 years, range $14-54$

b Number estimated on the basis of randomized stratified sampling in women giving birth in the Donostia University Hospital during the study period (see "Methods"). The origin of Chlamydia-positive women was individually collected after their detection in screening

c Prevalence was $9.6 \%$ (95\% Cl 5.6-16.0) (12/125) and $5.5 \%$ (95 \% Cl 3.8-8.0) (26/471) in women younger than 20 years and those aged $20-24$ years old, respectively

d Fisher exact test (two tailed)

e Age-adjusted prevalence was $1.0 \%(95 \% \mathrm{Cl} 0.8-1.2)$ for the entire group and was $0.8 \%(95 \% \mathrm{Cl} 0.6-1.0)$ and $1.9 \%(95 \% \mathrm{Cl} 1.3-2.5)$ for native women and foreign immigrants, respectively, Chi square $=20.54, P<0.001$

Perinatal transmission was detected in 10/91 (11 \%) neonates studied. Four of them were symptomatic and presented conjunctivitis.

\section{Discussion}

The present study was conducted in the immediate postpartum of women representative of the fullterm pregnant women in our region that attended public healthcare. The median age at delivery in the San Sebastián area was high, which may explain why the overall prevalence of infection was considerably lower than in pregnant women from central and northern European countries (1 vs. 2-3.9 \%) (Rours et al. 2011; Böhm et al. 2009; McMillan et al. 2006; Oakeshott et al. 2002; Kirk et al. 2008; Peuchant et al. 2015). As in most studies, the prevalence of infection in this study was higher in younger women. The prevalence of infection among women younger than 25 years $(6.4 \%)$ was closer to that found in the previously-mentioned European studies, showing figures somewhat higher than in Germany (4.5-5.7 \%) (Böhm et al. 2009) but somewhat lower than in France (7.9\%) (Peuchant et al. 2015), the United Kingdom (8.6-8.7 \%) (Oakeshott et al. 2002; Kirk et al. 2008), Eire (8.7\%) (McMillan et al. 2006) and the Netherlands (9.1\%) (Rours et al. 2011). The prevalence decreased sharply in older parturient women participating in this survey, which could partly be because women tend to have fewer partners as they get older. No N. gonorrhoeae infection was found in this large group, similar to the results recently obtained in the South of France in a group of 1004 pregnant women (Peuchant et al. 2015) and in Northern Italy among 2099 sexually active adolescents (Matteelli et al. 2016).
Information on the prevalence of C. trachomatis infection in the sexually-active general population in Spain is very scarce, since only one population-based study, limited to a town in the region of Asturias, has been conducted in the last decade (Fernández-Benítez et al. 2013). In that study, the prevalence of infection among sexuallyactive women younger than 25 years (4\%, range $2.8-$ $6.4 \%)$ was fairly similar to that observed in the present study. The prevalence of infection was higher in immigrant women as a whole, and especially in women of Latin American origin, who represent the main source of migration to Spain. These findings were probably related to the higher infection rates in their countries of origin (Cabeza et al. 2015; Pinto et al. 2011). Migration to Spain is a relatively recent event and, in studies conducted in other populations in Spain, has been considered an independent risk factor for C. trachomatis infection (Corbeto et al. 2010).

The study has some limitations. Because of the large number of pregnant women included in this study, no data on sexual activity were collected prospectively. Moreover, using nucleic acid amplification tests, the sensitivity of first catch urine specimens to detect $C$. trachomatis might be somewhat lower (up to $10 \%$ ) than that obtained from the vagina or cervix (CDC et al. 2014). Therefore the prevalence of $C$. trachomatis in women in Gipuzkoa could be slightly higher than that found in this study. However, first catch urine is an acceptable sample for the diagnosis of $C$. trachomatis urogenital infections in women (CDC et al. 2014), it is commonly used for C. trachomatis screening during pregnancy (Satterwhite et al. 2012; Böhm et al. 2009) and has been used in prevalence studies (Rours et al. 2011; McMillan et al. 
2006). In addition, although lower percentages of invalid results have been referred by other authors (Rockett et al. 2010) they did not test urine specimens from postpartum women, among which haematuric urines are common. The percentage of invalid tests in our study (4.4\%) was similar to that found in another study in parturient women from Brazil (Pinto et al. 2011) in which $3.7 \%$ of women were excluded because of bleeding during sample collection. Finally, although women were warned that urine collection should be first catch, we cannot ensure that it was the collected sample in all cases.

The most effective measure to control C. trachomatis infection is population-based screening in adolescents and young adults, but it is difficult to implement (ECDC 2014). An additional policy is screening in pregnancy. This form of screening is easy to implement and can achieve high coverage, since pregnant women usually make use of prenatal care and are usually willing to undergo diagnostic tests that could prevent adverse pregnancy outcomes. Pregnancy-based screening would detect infected women who are therefore at risk of pregnancy complications, and of transmitting the infection to their infants during delivery (Rours et al. 2011; Hammerschlag 2011; ECDC 2014; CDC et al. 2010). Most of these women are asymptomatic and their infection would remain undetected without screening. In this context, the high prevalence observed in young women in this study supports the need to recommend $C$. trachomatis screening in pregnant women $\leq 25$ years during their first gestational control visit in this region of Spain and the possible broadening of this strategy to women aged 25-29 years.

\section{Conclusion}

The results of this large study show that prevalence of $C$. trachomatis infection was high among pregnant women younger than 25 years (6\%). C. trachomatis infection represents a significant public health problem in this Southern European region. A gestational screening should be implemented in young women as part of antenatal care in Spain.

\footnotetext{
Abbreviations

C. trachomatis: Chlamydia trachomatis; Cl: confidence interval; HIV: human immunodeficiency virus; PCR: polymerase chain reaction; CMIA: chemiluminescent microparticle immunoassay; FTA-Abs: fluorescent treponemal antibody absorption; VDRL: Venereal Disease Research Laboratory; ECDC: European Centre for Disease Prevention and Control; CDC: Centers for Disease Control and Prevention (USA).

\section{Authors' contributions}

$L P, G C, A L$, and EPT conceived of the study, carried out its design and drafted the manuscript. LP and GC conducted the laboratory analysis. AL, IL, and LPMG contacted the women, organized sample collection, and performed the clinical histories. JK attended children of Chlamydia-positive mothers. All authors read and approved the final manuscript.
}

\section{Author details}

${ }^{1}$ Microbiology Department, Hospital Universitario Donostia, Paseo Dr Beguiristain s/n, 20014 San Sebastián, Spain. ${ }^{2}$ Obstetrics and Gynecology Department, Hospital Universitario Donostia, San Sebastián, Spain. ${ }^{3}$ Biomedical Research Centre Network for Respiratory Diseases (CIBERES), San Sebastián, Spain. ${ }^{4}$ Pediatrics Department, Hospital Universitario Donostia, San Sebastián, Spain. ${ }^{5}$ Department of Medicine Preventive and Public Health, UPV/EHU, San Sebastián, Spain.

\section{Acknowledgements}

This work was partially supported by a grant from the Fondo de Investigación Sanitaria (FIS PI10/02191). The authors thank Cristina Sarasqueta (Instituto de Investigación Biodonostia, San Sebastián, Spain) the help to perform the statistical analysis.

\section{Competing interests}

The authors declare that they have no competing interests.

Received: 18 August 2015 Accepted: 3 May 2016

Published online: 10 May 2016

\section{References}

Blas MM, Canchihuaman FA, Alva IE, Hawes SE (2007) Pregnancy outcomes in women infected with Chlamydia trachomatis: a population-based cohort study in Washington state. Sex Transm Infect 83:314-318

Böhm I, Gröning A, Sommer B, Müller HW, Krawczak M, Glaubitz R (2009) A German Chlamydia trachomatis screening program employing semiautomated real-time PCR: results and perspectives. J Clin Virol 46(Suppl 3):S27-S32

Cabeza J, García PJ, Segura E, García P, Escudero F, La Rosa S, León S, Klausner JD (2015) Feasibility of Chlamydia trachomatis screening and treatment in pregnant women in Lima, Peru: a prospective study in two large urban hospitals. Sex Transm Infect 91:7-10

Cates W Jr, Wasserheit JN (1991) Genital chlamydial infections: epidemiology and reproductive sequelae. Am J Obstet Gynecol 164:1771-1781

Centers for Disease Control and Prevention, Workowski KA, Berman SM (2010) Sexually transmitted diseases treatment guidelines, 2010. MMWR Recomm Rep 59(RR-12):1-110

Centers for Disease Control and Prevention, Papp JR, Schachter J, Gaydos CA, Van Der Pol B (2014) Recommendations for the laboratory-based detection of Chlamydia trachomatis and Neisseria gonorrhoeae. MMWR Recomm Rep 63(RR-2):1-19

Corbeto EL, Lugo R, MartrL E, Falguera G, Ros R, Avecilla A, Coll C, Saludes V, Casabona J (2010) Epidemiological features and determinants for Chlamydia trachomatis infection among women in Catalonia, Spain. Int J STD AIDS 21:718-722

European Centre for Disease Prevention and Control (2014) Chlamydia control in Europe: literature review. ECDC, Stockholm. doi:10.2900/16352. ISBN 978-92-9193-548-2

Fernández-Benítez C, Mejuto-López P, Otero-Guerra L, Margolles-Martins MJ, Suárez-Leiva P, Vazquez F, Chlamydial Primary Care Group (2013) Prevalence of genital Chlamydia trachomatis infection among young men and women in Spain. BMC Infect Dis 13:388

Haggerty CL, Gottlieb SL, Taylor BD, Low N, Xu F, Ness RB (2010) Risk of sequelae after Chlamydia trachomatis genital infection in women. J Infect Dis 201 (Suppl 2):S134-S155

Hammerschlag MR (2011) Chlamydial and gonococcal infections in infants and children. Clin Infect Dis 53(Suppl 3):S99-S102

Kirk E, Bora S, Van Calster B, Condous G, Van Huffel S, Timmerman D, Bourne T (2008) Chlamydia trachomatis infection in patients attending an Early Pregnancy Unit: prevalence, symptoms, pregnancy location and viability. Acta Obstet Gynecol Scand 87:601-607

Matteelli A, Capelli M, Sulis G, Toninelli G, Carvalho AC, Pecorelli S, Caruso A, Bonfanti C, Gargiulo F, Donato F, on behalf of the Clamigon Study Group (2016) Prevalence of Chlamydia trachomatis and Neisseria gonorrhoeae infection in adolescents in Northern Italy: an observational school-based study. BMC Public Health. 16(1):200. doi:10.1 186/s12889-016-2839-x 
McMillan HM, O'Carroll H, Lambert JS, Grundy KB, O'Reilly M, Lennon B, Collins C, Walsh TA, Geary MP, Cafferkey MT (2006) Screening for Chlamydia trachomatis in asymptomatic women attending outpatient clinics in a large maternity hospital in Dublin, Ireland. Sex Transm Infect 82:503-505

Oakeshott P, Hay P, Hay S, Steinke F, Rink E, Thomas B, Oakeley P, Kerry S (2002) Detection of Chlamydia trachomatis infection in early pregnancy using self-administered vaginal swabs and first pass urines: a cross-sectional community-based survey. Br J Gen Pract 52:830-832

Peuchant O, Le Roy C, Desveaux C, Paris A, Asselineau J, Maldonado C, Chêne G, Horovitz J, Dallay D, de Barbeyrac B, Bébéar C (2015) Screening for Chlamydia trachomatis, Neisseria gonorrhoeae, and Mycoplasma genitalium should it be integrated into routine pregnancy care in French young pregnant women? Diag Microbiol Infect Dis 82:14-19

Pinto VM, Szwarcwald CL, Baroni C, Stringari LL, Inocêncio LA, Miranda AE (2011) Chlamydia trachomatis prevalence and risk behaviors in parturient women aged 15 to 24 in Brazil. Sex Transm Dis 38:957-961
Rockett R, Goire N, Limnios A, Turra M, Higgens G, Lambert SB, Bletchly C, Nissen MD, Sloots TP, Whiley DM (2010) Evaluation of the cobas 4800 CT/NG test for detecting Chlamydia trachomatis and Neisseria gonorrhoeae. Sex Transm Infect 86:470-473

Rours GI, Duijts L, Moll HA, Arends LR, de Groot R, Jaddoe VW, Hofman A Steegers EA, Mackenbach JP, Ott A, Willemse HF, van der Zwaan EA, Verkooijen RP, Verbrugh HA (2011) Chlamydia trachomatis infection during pregnancy associated with preterm delivery: a population-based prospective cohort study. Eur J Epidemiol 26:493-502

Satterwhite CL, Gray AM, Berman S, Weinstock H, Kleinbaum D, Howards PP (2012) Chlamydia trachomatis infections among women attending prenatal clinics: United States, 2004-2009. Sex Transm Dis 39:416-420

World Health Organization (2012) Global incidence and prevalence of selected curable sexually transmitted infections-2008. http://www.who.int/iris/ handle/10665/75181

\section{Submit your manuscript to a SpringerOpen ${ }^{\circ}$ journal and benefit from:}

- Convenient online submission

- Rigorous peer review

- Immediate publication on acceptance

- Open access: articles freely available online

- High visibility within the field

- Retaining the copyright to your article 